\title{
Nasal Cavity and Paranasal Sinus Cancer by AJCC v7 Stage
}

National Cancer Institute

\section{Source}

National Cancer Institute. Nasal Cavity and Paranasal Sinus Cancer by A/CC v7 Stage. NCI

Thesaurus. Code C91255.

A term that refers to the staging of nasal cavity and paranasal sinus carcinoma according to the American Joint Committee on Cancer, 7th edition. 